\title{
MÚSICA HOLOFRACTAL: UNA CONEXIÓN SEMIÓSICA ENTRE LA MÚSICA Y LA FÍSICA CONTEMPORÁNEAS
}

\section{Eufrasio Prates}

\author{
IESB - Instituto de Educação Superior de Brasília
}

There is always the possibility of proving any definite theory wrong; but notice that we can never prove it right.

Richard Feynman (1992: 157)

Physis es la palabra griega para naturaleza, que da origen al término física. El siglo XX presenció el surgimiento de una nueva percepción de la naturaleza, a través de la física cuántica. De este modo, la nueva física alcanzó y unió dos universos invisibles y supuestamente distantes: las partículas subatómicas y el espacio sideral. Esto llevó, también, a sorpresivos descubrimientos, tan paradójicos e imprevisibles como el propio comportamiento de la naturaleza, evidenciando la imposibilidad de hacer cualquier afirmación definitiva o predicción absoluta a este respecto. Las teorías más importantes nacidas bajo esta visión cuántica tienen en común el concepto de holos, que significa 
totalidad, integralidad, el todo. Desde los años 60 , algunos físicos comenzaron a referirse al paradigma holográfico o holonómico, dando énfasis a la trascendencia de la interrelación entre la parte y el todo (Prates, 1999: 52). Todos estos cambios estructurales abrieron nuevos campos de investigación, y en especial uno, al parecer extremamente provechoso: la ciencia del caos.

\section{INTERSEMIOSIS FÍSICA-MÚSICA}

La definición de la palabra caos, que para el sentido común significa el opuesto categórico de orden, inmediatamente nos coloca en una situación difícil: como Floyd Merrell (1998) señala en su libro Simplicity and complexity existe una especie de orden intrínseco al propio caos. En realidad, el supuesto desorden del caos no es más que nuestra incapacidad de comprender los sistemas no periódicos, que caracterizan a la naturaleza y a la sociedad como, por ejemplo, la turbulencia de los fluidos, las variaciones en economía, las transformaciones meteorológicas, la explosión demográfica y un sin número de fenómenos que no se encuadran en las famosas condiciones normales de temperatura y presión -inexistentes en el universo-. En sus antiguas raízes griegas, el término chaos significaba origen o abertura originaria de donde viene todo, o sea, indeterminado. Será sólo con el sentido romano cuando esta palabra llegará a ser conocida como desorden, en oposición a kosmos u orden, lo que, en el contexto de complejidad en el que vivimos, llega a ser anacrónico.

Permítanme tomar un ejemplo simple. La segunda ley de la termodinámica, que dice que el universo camina, sin retorno, en dirección a estados mucho más caóticos, es frecuentemente explicada al lego a través del experimento en el que se mezclan el agua y la tinta. Es posible probar la entropía de este sistema por el hecho de que estos componentes líquidos nunca volverán, «naturalmente», a su estado original. No obstante, este mismo experimento puede servir para probar y explicar una nueva organización, orgánica, homogénea y, por tanto, entálpica. Fue éste uno de los primeros problemas enfrentados por algunos de los científicos de la década de los sesenta. En los estudios embrionarios sobre sistemas no periódicos encontraremos nombres como Benoit Mandelbrot y Christopher Scholz, creadores de las nuevas matemática y geometría, mucho más próximas a la naturaleza y a la 
sociedad. Esos sistemas son también conocidos como no lineares, por su imprevisible desarrollo y por la dependencia sensible de sus condiciones iniciales. El célebre efecto mariposa ejemplifica bien esto al proponer que el aire provocado por las alas de una mariposa, en algún lugar distante del planeta, puede desencadenar un huracán. Esto nos conduce a una definición técnica de caos: fenómeno altamente dependiente de sus condiciones iniciales (Lorenz, 1996: 39).

Como el mundo siempre había rechazado el comportamiento apoyado en las reglas euclidianas, estos científicos iniciaron la construcción de un nuevo camino, la geometría fractal, para describir la complejidad de nuestros litorales, las microirregularidades en superficies metálicas, la porosidad de las rocas llenas de petróleo, etc. Mandelbrot, abrazando una aproximación relativista en el profundo estudio de las dimensiones, llegó a la conclusión de que, para una mejor comprensión, la naturaleza exige el tránsito a través de las fracciones entre el punto y la línea, la línea y el plano, el plano y el volumen. ¿Cómo deberíamos calcular la cantidad de petróleo que hay en una piedra porosa? Descubriendo un número entre la segunda y tercera dimensiones que se aproxime a este estado real y complejo. Algo como, por ejemplo, 2.4 dimensiones. Basado en esta nueva lógica, es posible calcular el grado de irregularidad o fragmentación de un determinado fenómeno. A partir de esta segmentación, esta fractura, Mandelbrot creó la palabra fractal en 1975.

Una aproximación más reciente a la ciencia fractal se sustenta en las ideas de Edward Lorenz, Mitchell Feigenbaum, Stephen Smale y David Ruelle, que se desarrollaron a partir de la observación de fenómenos naturales, designando una concepción mucho más compleja de la naturaleza, basada en una aproximación más simple. Recolectando datos sobre la turbulencia de fluidos viscosos o del cambio climático, y descubriendo ecuaciones que representan sus modelos complejos y ocultos, dichos científicos llegaron a conclusiones que permitieron la construcción de conceptos como atractor extraño, no linearidad, caprichosidad $y$ autosimilaridad. Este último muestra que hay en la naturaleza algún tipo de interelación fuerte entre lo grande y lo pequeño. Hans Lauwerier (1991: XI) afirma que: «un fractal es una figura geométrica en la cual un mismo motivo se reproduce a sí mismo en escala siempre decreciente», a pesar de hacer mención al hecho de que la definición original de Mandelbrot se refiere a un concepto más estricto y complejo de dimensión fractal. Buenos ejemplos de la singularidad e importancia de esta geometría orgánica son la Torre Eiffel —construida sobre una fina, pero 
resistente estructura, autorreproducida en escala fractal-, o los pulmones humanos -que fractalmente compactan una superficie que podría ocupar un área mayor a una cancha de tenis-.

Esas nuevas teorías - potencialmente sumarizadas en conceptos como holonomía, paradojicalidad, fractalidad, atemporalidad, no causalidad (Prates, 1999: 53) - sintetizan un gran paradigma que denominé holofractal. Recordando a Feynman (1992: 157), en la cita de apertura de este artículo, se puede tratar de teorías no absolutamente correctas. Sin embargo, éstas evocan una visión de mundo de mayor proximidad con la naturaleza, como hace mucho tiempo no se veía.

Desde luego, los modelos sirven para muchas cosas. Algunos de ellos son potencialmente peligrosos, por hacer un mal empleo de su reduccionismo en una realidad compleja. Es el caso del positivismo racionalista que, desafortunadamente, aún se difunde por nuestras instituciones de investigación. Sin embargo, un modelo bueno sería el aplicar estos conceptos y relaciones internas en diferentes campos del conocimiento. Dicha tarea es la que yo he venido desarrollando durante diez años: construyendo conexiones paradigmáticas entre la música y la física; aplicando, en suma, la intersemiosis.

\section{DE LA NATURALEZA A LA CULTURA}

la experiencia estética [...] sugiere que los códigos de los cuales parte podrían ser sometidos a segmentación sucesiva.

Umberto Eco (1976: 227)

Es ampliamente sabido que la música de Johann Sebastian Bach está alta y matemáticamente organizada. Esta cualidad nunca ha generado críticas negativas acerca de su música, ya que siempre ha estado claro que en ella priman las características estéticas. Por el contrario, en ciertos trabajos dodecafónicos contemporáneos encontramos un modo opuesto de abordar este problema. El método serialista intentó anular la tradición mediante la mecanización del proceso de composición y, pese a sus avances tecnológicos, rápidamente mostró ser excesivamente rígido, dando como resultado una música fría e inhumana. Hoy en día necesitamos encontrar un método equilibrado que evidencie las ideas centrales de nuestro tiempo, sin abandonar el insustituible carácter humano-estético. Una adecuada res- 
puesta a esto es el uso del conocimiento semiótico como un puente entre la naturaleza y la cultura. En otras palabras, la semiótica parece ser el instrumento que contribuirá a llenar el vacío dejado por el abandono de métodos tradicionales de composición.

Lejos de hacer definiciones precipitadas, veo a la semiótica como el sendero de intuición a través de los lenguajes, con la finalidad de entender la compleja multiplicidad de estratos que conforman el significado de un fenómeno. Si esto parece ser una forma inusual de ver la ciencia, debemos recordar que las exploraciones más creativas de nuevos campos hallan su camino a la luz del concepto peirciano de abducción. Con este término, Peirce designó al instintivo y sugestivo razonamiento, típico de la irrupción de insights (Sebeok, 1991: 9; Peirce, 1995: 220-221). De hecho, el método abductivo es descrito en palabras como percepción, prueba, y error y experimentación.

Esta visión, por otro lado, puede arriesgarse a perder su riqueza, si la dimensión comunicacional se deja lado. Nunca debemos olvidar que la música se hace para ser escuchada por otros. Obviamente, ningún compositor contemporáneo se engaña en relación a la comprensión de su trabajo, lo que no implica la ausencia de preocupación en el uso de los códigos. Aunque Mukarovsky (1981: 124) no esté equivocado acerca de la autonomía del signo estético, esto no libra a la creación poética de su papel comunicacional.

Esta señal de alerta se dirige a ciertos experimentos de música fractal, nacida de una aproximación extremadamente matemática. Por supuesto, en la producción de este tipo de arte, son necesarios muchos cálculos para trabajar con parámetros fractales. Las computadoras son importantes herramientas para administrar el amplio abanico de ecuaciones fractales. No obstante, es peligroso creer que éstas puedan solucionar problemas estéticos. Tal proceso de automatización debe manejar, exclusivamente, el desarrollo del repertorio y la manipulación del sonido. Todas estas tareas deben seguir un plan muy bien organizado, lo cual, algunas veces, es olvidado por los compositores fractales. Cualquier persona puede fácilmente alimentar un software de música fractal con algunos números al azar y generar una secuencia de sonidos extraña o sumamente redundante. La distancia entre este resultado rígido y lo que llamamos música requiere de la habilidad de un músico, quien posee el conocimiento estético necesario para transformar sonidos en un fenómeno complejo, significativo. 
El juego entre forma y elemento, entre el todo y la parte, es precisamente el primer proceso para ensamblar nueva música y nuevos paradigmas. En efecto, el compositor de música fractal debe reunir todos los conceptos citados con las finas técnicas de muestreo, cálculo, edición y secuencia de sonidos. Pero todo esto no basta: sin una especial atención al lenguaje —el puente entre la realidad concreta y la semiosfera- este proceso puede llegar a ser esquizofrénico, dividido en teoría y práctica, en técnica y estética. Únicamente una aproximación semiótica, explícita o no, puede evitar este serio problema. Y ello, porque una postura crítica es indispensable para materializar ideas neoparadigmáticas en prácticas comunicativas. De ello, se preocupó Peirce en el montaje del Diagrama de las Ciencias, organizando las relaciones entre las tres ciencias normativas: la Lógica —que para él es sinónimo de Semiótica-, la Ética y la Estética (Santaella, 1994: 136). El método debe someterse al bien común que, a su vez, debe nacer de una admiración íntima, afectiva y auténtica. A partir de ahí transcurrirán las principales líneas de los experimentos en el campo de la composición holofractal relatadas aquí. Con el objetivo de exponer los primeros resultados de ese trabajo, presento a continuación un análisis de mi obra, titulada coVeranS.

\section{UN ESTUDIO DE CASO EN MÚSICA HOLOFRACTAL}

Estos 3min y 20seg de composición de música experimental fueron finalizados en 1996. Su proceso de creación puede dividirse en tres partes, para propósitos didácticos: diseño del proyecto (terceridad), tratamiento fractal de los sonidos (primeridad) y edición de las muestras sonoras (segundidad). coVeranS fue concebida con la idea de vincular lo «sintético» con lo «orgánico», interconectando cultura y naturaleza a través de un puente semiósico.

Por las razones discutidas anteriormente, esta pieza evita la forma discursiva causal, porque semiosis y fractales son fenómenos complejos, no lineales e impredecibles. Usé una nueva forma de organización, desarrollada por compositores contemporáneos, particularmente por H. J. Koellreutter, quien la llamó forma sinerética. Koellreutter (1990: 120) describe sinéresis como el «resultado de un proceso de percepción transracional que produce el sentido de unidad, integrando los elementos en un todo». Para organizar música 
sineréticamente, es necesario, primero, disponer los signos musicales seleccionados en el tablero para crear un bosquejo de la pieza. Después de que llegó a visualizarse la totalidad, fueron diseñadas múltiples interconexiones entre los elementos, seguidos por la selección de una versión temporal que se confronta con el esquema de propósitos estéticos. Para este trabajo fue utilizado el software de secuenciamiento de archivos de audio $D E C K$, un multitracker de ondas digitales.

Tomé la decisión de elegir dos elementos básicos como los principales signos originales: un sonido sintético de percusión, imitando al tambor, y una voz humana, diciendo las palabras: «geometría fractal». Esta selección fue hecha para construir un repertorio de alto contraste, donde la variación pretendió llenar el espectro entre la percusión sintética y los sonidos de la voz orgánica. Ambas unidades fueron digitalizadas y grabadas en una computadora con los nombres snare y fracvoc. Éstas fueron las únicas fuentes para generar todos los otros sonidos mutantes de esta pieza, al principio llamada SnareVoc y, finalmente, denominada coVeranS.

Ése es el punto en el que los parámetros fractales entran en escena. Para crear nuevos sonidos a partir de estas fuentes, utilicé un programa de computación que, a diferencia de los tradicionales softwares de música fractal, penetra las entrañas del sonido. Investigando en Internet, descubrí que todas las referencias halladas están basadas en una secuencia de números tocada por el sintetizador MIDI. Tal abordaje, aunque interesante en ciertos sentidos, ignora las dimensiones internas del sonido, considerando únicamente parámetros macro y sencillos como duración, frecuencia y, en algunos casos, intensidad. Además de que este tipo de música es, la mayoría de las veces, muy redundante, severo y mecánico, a causa de su intrínseca dificultad en trabajar con estructuras y formas globales. Efectivamente, este tipo de música se vincula generalmente a los sonidos prefabricados en sintetizadores, lo que realmente empobrece los resultados.

Por esta razón preferí trabajar con las microdimensiones de la música. SoundHack, el software desarrollado por Tom Erbe en 1995, ofrece una elaboración de sonido digital que contempla la mutación espectral, dinámicas espectrales y convolución, entre muchas otras herramientas de procesamiento de las entrañas de los sonidos. Por otro lado, esto abre una puerta para el acceso de parámetros que serán sometidos a funciones de mutación interna, encarnando la idea de fractalidad, por ejemplo, la relación de iteración en el espectro de onda y tiempo. 
Para crear los sonidos básicos de coVeranS fue necesario, después de escoger un sonido de origen y un sonido de llegada, definir parámetros con el objetivo de establecer la forma en que estos sonidos deberían interactuar para producir un descendente mutante. Al igual que en el embarazo humano, pasé el período de procesamiento pensando en las características que tendría el nuevo sonido. Pese a que se usó un PowerPC Macintosh, con un rápido procesador RISC, en ocasiones un sonido de 38 segundos tomó más de cien horas de trabajo. Sorprendentemente, no fue inusual obtener resultados raros y fantásticos. Otras veces, se generó un archivo de absoluto silencio. Esto sucedió debido a que existe una fuerte relación entre este proceso fractal y el «efecto mariposa»: dependencia altamente sensible de las condiciones iniciales. Es notable cómo los sonidos transformados se presentan, paradójicamente, altamente contrastantes y semejantes a sí mismos. Esta insólita característica refleja la interrelación natural y orgánica entre la parte y el todo, tal como se generan todos los pequeños sonidos a partir de la muestra principal o de la intermutación entre los sonidos derivados mutantes. Bajo la lógica fractal, fue realizada una derivación sobre otra, transladando la interrelación parte-totalidad.

Este proceso, más rico que los comunes, permite la manipulación del propio sonido, enfatizando la función semiótica humana en la composición de estos pequeños elementos sonoros en una totalidad que constituye un sentido estético. Con el mencionado proceso de prueba y error, típico del pensamiento abductivo peirceano, fueron necesarios un par de meses para producir las variaciones y transformaciones que, simultáneamente, fueron trabajadas como partes del ensayo musical como un todo. Las líneas directrices de coVeranS han sido tejidas en forma de red —como la araña que busca el equilibrio entre técnica e intuición-.

Cada nueva posición de los sonidos en la pieza fue sometida a un ordenamiento no lineal, reforzando la imprevisibilidad y no causalidad de la composición. Como de un sonido ascendiente (sonido-madre) puede surgir después de sus descendientes (sonidos-hijos), se evitó la relación de causa y efecto. Asimismo, la complejidad de estos sonidos puede impedir la comprensión de esta música, especialmente por parte del oído racionalista. Sin embargo, es bueno no olvidar que estamos tratando con nuevos conceptos transracionales. Intuición es la clave para pasar del entendimiento a la comprensión.

Inspirado en la fenomenología triádica peirceana, nuestra segundidad se encuentra en el refinamiento de pruebas iniciales, nacidas bajo el 
proceso de mutación. El esfuerzo se emprendió a través del uso de otro programa llamado SoundEdit, que proporciona una buena variedad de herramientas de acabamiento. Esta fase fue extremadamente delicada, pues aplicar efectos y filtros en un sonido puede fácilmente destruir su «personalidad»y, peor que eso, puede desvincularlo de sus ancestros fractales. Los instrumentos de edición más usados fueron el equalizador, normalizador, pitch shifter, reverser, enveloper y bender.

Este software resultó muy útil para invertir algunos sonidos, así como para cortar y pegar partes de sonidos y crear efectos en un montaje de tipo cubista, quebrar la temporalidad lineal de la música, hasta en sus unidades más básicas. Esto intermedió el proceso de ida y vuelta entre la primeridad de las cualidades elementales del sonido y la terceridad de las relaciones simbólicas. Por esta razón, hubo un proceso inter-reflexivo y no lineal de creación, partiendo de unidades sonoras básicas hasta la macroestructuración y viceversa.

Para finalizar esta rápida exposición del ensayo: música holofractal, es conveniente mencionar una postura especial, necesaria para una fructífera experiencia auditiva. La mayor parte de las dificultades en escuchar música nueva no parte de la poca proximidad de los oyentes frente a ésta, ni tampoco de su ignorancia acerca del nuevo cuadro técnico, sino, principalmente, del profundo y arraigado prejuicio, heredado, y hoy reforzado por las estrategias capitalistas de los medios de comunicación de masas.

En lugar de rehusar lo nuevo por su extrañeza, sería deseable que se asumiera una posición intuitiva, preparándose para una experiencia estética. Después de eso, será más fácil ponerse conscientemente en el lugar de la mente creativa-interpretante, la única capaz de trazar la trayectoria entre la naturaleza y la cultura sobre una pieza de música intencionalmente incompleta.

\section{Referencias bibliográficas}

Eco, U. (1976). Tratado geral de semiótica. São Paulo: Perspectiva. Eco, U. \& SebeoK, T. (1991). O signo de três. São Paulo: Perspectiva. FeynMAN, R. (1992). The Character of Physical Law. London: Penguin Books. KoEllReutTer, H. J. (1990). Terminologia de uma nova estética da música.

Porto Alegre: Novas Metas. 
LAUWERIER, H. (1991). Fractals: Images of Chaos. London: Penguin Books.

LORENZ, E. N. (1996). A essência do caos. Brasília: Edunb.

Merrell, F. (1998). Simplicity and Complexity: Pondering Literature, Science and Painting. Michigan: University of Michigan Press.

MuKAROVSKY, J. (1981). Escritos sobre estética e semiótica da arte. Lisboa: Estampa.

PeIRCE, Ch. S. (1995). Semiótica. São Paulo: Perspectiva.

Prates, E. (1999). Passeio-relâmpago pelas idéias estéticas ocidentais. Brasília: Valci.

Santaella, L. (1994). Estética: de Platão à Peirce. São Paulo: Experimento. 